\title{
Modelling of a Vacuum Metallization Patterning Method for Organic Electronics
}

\author{
Thomas Cosnahan ${ }^{a}$, Andrew A.R. Watt ${ }^{a} \&$ Hazel E. Assender ${ }^{a}$ \\ a Department of Materials, University of Oxford, 16 Parks Road, Oxford, OX1 3PH, UK \\ hazel.assender@materials.ox.ac.uk
}

\begin{abstract}
The high throughput roll-to-roll patterning of metal thin films could be used to create organic functional devices. In this paper we present research into the compatibility of an invacuum selective metallization technique, which uses a sacrificial oil to define the metal electrode pattern for functional devices. The exact mechanism of the sacrificial oil masking, pattern definition and oil vaporization, due to the radiant heating from the thermal evaporation source, is described from previous research and experimental findings. The thermal modeling of this process develops further the requirements for the masking oil. It is found that in this particular system the oil thickness must be $1.63 \pm 0.31 \mu \mathrm{m}$ to match the radiative energy, and not be evaporated prior to leaving the metallization zone or to remain, as excess, after metallization is complete. The temperatures required are low and compatible with heat sensitive functional organic materials. The definition of the metal pattern edges is found to be like the liquid oil but thermal modelling still supports the theory of the oil vapor having the largest masking effect and concludes that the pattern definition quality does not depend on the sacrificial oil masking or vaporization effects but on the flexography patterning.
\end{abstract}

Keywords: flexography; metallization; roll-to-roll; organic transistor; vacuum; patterning 
Funding: This work was supported by the Engineering and Physical Sciences Research Council 


\section{Introduction}

Selective metallization is an industrial process that has been used to make decorative packaging and holograms [1-3]. It is a cost-effective metal thin film patterning process, which does not use environmentally hazardous etchants or require copious energy. Since the original patent expired in 2001 it has been adopted by several commercial businesses $[3,4]$. If compatible with organic electronics it would be a viable method for the high throughput production of functional devices. Not only would this industrially mature high throughput process be able to create large areas of functional devices but there would be improvements on the thin film's properties in terms of low thickness and roughness, and high conductivity compared with solution based processes such as screen printing and inkjet.[5-7]

This paper will study the selective metallization process to show that it is compatible, in terms of process conditions, with organic devices. The thermal mechanism of the process will be described showing that it would not damage the functional device and that this process has a wide range of applicability. The properties, such as conductivity and roughness, of the patterned metal are also presented to show the advantages of metal thin film patterns made with this technique over other metal patterning techniques for organic electronics. This in-line patterning technique has the promise to replace the state-of the art deposition method for best quality conductive contacts: that of vacuum evaporation or sputtering with shadow masking, as solid-state shadow masking is unattractive for in-line processing.

The selective metallization process is illustrated in Figure 1. In high vacuum $\left(<10^{-4} \mathrm{mbar}\right)$, on a rotating process drum a substrate has a pattern of oil applied to it, in this case using a 
soft stamp flexography technique. Next, the oil-patterned substrate is rotated past the metal evaporation source, where the metal vapor condenses onto the substrate but it does not adhere to the oiled regions; this creates the metal pattern. Simultaneously the sacrificial oil is heated and evaporated off the substrate, to be pumped out the chamber, resulting in areas with and without metal deposition.

The masking oil evaporates due to the radiant heating of the substrate as it passes the metallization source. Akin to additive photolithography, the oil acts as the photoresist and masks the negative pattern regions, so the removal of the oil is analogous to the lift off step, hence it is coined 'thermal lift off' here. To avoid metalizing the masked substrate areas, the oil volume must not be removed before the substrate has left the metallization zone. Conversely the sacrificial oil must be completely removed from the substrate once it has left the metallization zone. This balance, in the thermal lift off process, is the crucial to prevent unwanted blurring of the metal pattern's edges or any excess oil affecting the underlying substrate (which is a functional organic with sensitive chemistry), or requiring further oil removal steps.

This thermal balance has several factors which can be manipulated to optimize the process:

- The oil volume transferred to the substrate by the oil patterning process;

- The physical properties of the sacrificial masking oil which control the vaporization behavior ;

- The amount of radiative heating seen by the oil in the thermal evaporation process, which includes the process conditions and system geometry. 
The oil volume transferred to the substrate will determine the liquid oil thickness and homogeneity and the patterning process will define this liquid volume's line edges. The oil patterning process in this work has been performed using a lab scale flexographic deposition process. The custom-built system was optimized by varying the pressure and print speed to minimize pattern edge roughness. Other methods include oil vapor jets[2], inkjet[8] and other roller impression transfer techniques[1,9].

The oil is selected to be chemically inert and compatible with the vacuum environment and flexography printing, which is a versatile printing technique with a wide viscosity range of 0.05-0.5Pa.s .[10] Also the sacrificial masking oil must have the physical properties for preventing metallization onto the substrate and suitable properties to allow it to evaporate off the substrate in the lift off step of the selective metallization process.

Pagani, et al. [11] investigated the efficiency of different types of sacrificial oils used for the selective metallization process. Using a model of the vaporized oil cloud 'shielding' the substrate from the metal vapor, they equilibrated the ideal vapor pressures of the oil and metal vapors to find an expression defining the physical properties that control the shielding oil vapor cloud layer's depth. Reducing this vapor cloud layer thickness would reduce oil consumption and the authors also suggested that a thinner oil vapor shield layer would produce a more 'precise' print, taken to mean greater print fidelity with high resolutions. Their model showed that the oil's vapor pressure, which acts against the aluminum's vapor pressure, is inversely proportional the vapor thickness. Relatedly, a high latent heat of vaporization makes the thermal lift off process more effective, as the radiative energy required for vaporization is increased, which protects the substrate, or functional 
device, from damaging temperature increases, given a constant pressure and temperature during the liquid-vapor phase change.

This can be summarized by writing the Clausius-Clapeyron equation in terms of the thermal lift off process temperature $\left(T_{\text {Process }}\right)$, the oil's specific volume $\left(v_{\text {Oil }}\right)$ and latent heat $\left(H_{\text {Oil }}\right)$. The radiative heating of the liquid oil causes the vaporization phase change, over a temperature change $\left(d T_{\text {Radiative }}\right)$ and a pressure change $\left(d P_{\text {Oil }}\right)$ in the oil:

$$
\frac{d P_{\text {Oil }}}{d T_{\text {Radiative }}}=\frac{H_{\text {Oil }}}{T_{\text {Process. }} v_{\text {Oil }}} \cdot\{1\}
$$

In order to reduce the term $T_{\text {Process }} . v_{\text {Oil }}$ which will protect the substrate and reduce oil consumption, while maximizing $d P_{\text {oil }} / d T_{\text {Radiative }}$ to maximize the oil masking pressure for a given radiative heating amount, means increasing the oil's latent heat.

The latent heat of the oil is determined by the physical chemistry of the oil, such as the molecular weight, which can act to contain the vapor cloud close to the liquid oil patterned on the substrate. A large molecular weight not only reduces a vapor molecule's mean free path but can also introduce more inter-molecular bonding force.

In this work, an industry standard Krytox ${ }^{\circledR}$ oil [12] was used which is a high molecular weight perfluoropolyether. This chemical structure is well suited to vacuum systems, it is inert and has a viscosity suitable for flexography printing. Also the fluorinated chemistry will have instantaneous dipole-induced intermolecular forces, which are attractive and create a positive internal pressure. In the aforementioned model, the oil's vapor was 
modeled as an ideal gas, whereas behaving as a real vapor, as per the Van der Waal equation:

$$
p=\frac{n R T}{V-n b}-a \frac{n^{2}}{V^{2}}
$$

where $p, n, R, T$ and $V$ is pressure, molar amount, Boltzmann constant, absolute temperature and volume respectively. The term $a$ is the Van der Waal constant, related to the internal pressure $\left(\pi_{T}\right)$ by the definition:

$$
\pi_{T}=a \frac{n^{2}}{V^{2}}
$$

The constant $a$ is $2.25 \mathrm{~Pa}^{\mathrm{m}} \mathrm{m}^{6} \cdot \mathrm{mol}^{-1}$ for the perfluoropropyl methyl ether repeat unit of the particular oil in question [13]. The constant $b$ is another constant taking account the molecule's volume. Because of the positive internal pressure due to the attractive force, the intermolecular forces will act to constrain the vaporized molecules more-so than previously implied. The Van der Waal's constants suggest the real vapor behavior will be better suited to the process than a modelled ideal gas due to the oil's chemistry, as the oil vapor will be more localized to the oil liquid pattern region on the substrate.

The radiative heating of the oil as it passes over the metallizing source can be used to calculate the optimal volume of liquid oil, to prevent metallization but with complete removal of the liquid oil. This is described in the methods section and assumes that the oil vapor acts as a mask above the liquid oil so that no aluminum vapor condenses onto the 
liquid oil, but only the radiative energy of the evaporation source contributes towards the thermal lift off process. This assumption is discussed in the following sections. 


\section{Materials and Methods}

The volume of liquid oil required for effective masking was modeled by calculating irradiation heat $(Q)$ which is the radiative energy per unit square:

$$
Q\left(W m^{-2}\right)=A \varepsilon_{\text {source }} \varepsilon_{o i l} \sigma\left(T_{A l}^{4}-T_{O i l}^{4}\right)
$$

where $A$ is the geometric view factor of the oil on the process drum as it passes over the evaporation boat, $\varepsilon_{x}$ is the emissivity of $x, \sigma$ the Stefan-Boltzmann constant, $T_{A l}$ the temperature of the aluminum thermal evaporation source, and $T_{\text {oil }}$ the temperature of the oil as it enters the metallization zone. The thermal radiation energy $(E)$ seen by oil as it passes the source is:

$$
E\left(\mathrm{Jm}^{-2}\right)=Q . \frac{\text { metalization zone length }}{\text { web speed }}
$$

Therefore the oil thickness $(t)$ required to match this thermal energy is calculated by dividing by the specific and vaporization heats:

$$
t=\frac{E}{\rho\left(C_{p} \cdot \Delta T_{o i l}+C_{V}\right)}
$$

where $C_{P}$ and $C_{V}$ are the specific heat and vaporization heat respectively and $\rho$ is the density at the vaporization temperature and $\Delta T_{\text {oil }}$ the change in temperature of the oil from ambient liquid state to the vaporization temperature.

A series of samples were printed with aluminum stripes perpendicular to the web direction, using a rotating relief stamp, i.e. flexography printing plate, made of poly dimethyl siloxane 
(PDMS, purchased from Dow Corning) in the standard 10:1 weight ratio of base and curing agent. The rotating stamp, supported on a steel cylinder and foam backing (stiffness = $18.3 \mathrm{kPa}$ ) was impressed, using hanging dead weights, axially against the process cylinder which was wrapped with the polyethylene terephthalate (PET) substrate. Inking of the raised areas of the stamp was achieved by initially passing an oil saturated membrane through the plate/process drum nip, mimicking an anilox system; ensuring sufficient ink volume is available to be transferred for complete inking of the printing plate. 24 hour swell tests, as per Lee et. al.'s method showed no swelling of the PDMS due to the sacrificial oil.[14] Optimal printing pressure and speed were used to achieve good print quality and ensure adequate registration; image analysis [15] was performed to quantify the print quality in terms of the printed line width and line edge roughness by identifying the stripe edges and performing pixel position calculations, see reference for more detail. The deposited patterns were imaged with optical microscopy (Leica M165 C) at 10x magnification.

The printed pattern contained 10 metal lines with a nominal (plate) linewidth of $150 \mu \mathrm{m}$, of which the nine oil masked regions were analyzed and the line width and edge roughness data averaged. Electrical characterization was performed with a four point probe and thickness measurements were made with MicroXam optical profiler, with five repetitions averaged to give the reported values. Slight deviations in data are expected to be due to print pressure variance due to equipment imperfections and operational error with analysis equipment. 


\section{Results}

Equation 4 shows the importance of controlling the source temperature accurately, as the radiative energy scales to the fourth power. Maximizing the source temperature will ensure the oil is completely removed, but, as with all thermal evaporation processes damage to the substrate limits the safe operating temperature, which for functional organics is less than $100^{\circ} \mathrm{C}$ as a general rule, but could be even lower.

Table 1: The values used for calculating the required liquid oil thickness to match the radiative energy as per Equations 3-5. These values are calculated from the system geometry or DuPont Krytox ${ }^{\circledR}$ sales literature unless otherwise referenced.

\begin{tabular}{|c|c|}
\hline View factor & 0.5 \\
\hline Emissivity of the source & $0.04[16]$ \\
\hline Emissivity of the oil & $0.03[17]$ \\
\hline \multicolumn{2}{|l|}{ Stefan-Boltzmann Constant $\left(\mathrm{Wm}^{2-}\right.$} \\
\hline $\left.\mathrm{K}^{-4}\right)$ & $5.67 \times 10^{-8}$ \\
\hline Temp Source $(\mathrm{K})$ & $1250[18]$ \\
\hline Temp Oil (K) & 293 \\
\hline Irradiation $\left(\mathrm{W} \cdot \mathrm{m}^{-2}\right)$ & $1.45 \times 10^{3}$ \\
\hline Web speed $\left(\mathrm{m} \cdot \mathrm{s}^{-1}\right)$ & 0.04 \\
\hline Process zone length (m) & 0.31 \\
\hline Radiation seen by oil $\left(\mathrm{J}^{\prime} \mathrm{m}^{-2}\right)$ & $3.81 \times 10^{2}$ \\
\hline Oil Density $\left(\mathrm{kg} \cdot \mathrm{m}^{-3}\right) @ 62.5^{\circ} \mathrm{C}$ & 1799 \\
\hline Oil Vaporization enthalpy ( $\left.\mathrm{kJ} \cdot \mathrm{kg}^{-1}\right)$ & 37.78 \\
\hline
\end{tabular}




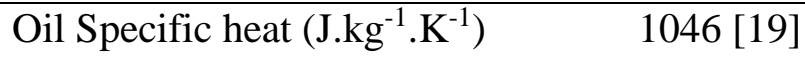

Oil Temperature Change (K) $\quad 42.5$

Given the typical values, shown in Table 1, the thickness of the liquid oil required is $1.63 \pm 0.31 \mu \mathrm{m}$ to match the radiative energy, which matches the order of magnitude of the thickness of oil deposited by the flexography print system, which is estimated to be $7.6 \pm 1.5 \mu \mathrm{m}$, assuming the volume of oil transferred at the contact of the impression drum and printing plate is a half, which is the maximum for high print speeds. This required liquid oil thickness is specific to the system, and would change proportionally with other view factors.

Figure 2 shows an example of the cross direction aluminum thin film stripes, patterned onto PET at $2.4 \mathrm{~m} \cdot \mathrm{min}^{-1}$ with nip line pressure of $50 \mathrm{Nm}^{-1}$. The pattern line edge roughness between the stripes, could translate into critical, shorting, defects for source/drain contacts of a transistor. The stripe edge's root mean square (RMS) roughness for this print was found to be $9.0 \pm 1.3 \mu \mathrm{m}$ and the peak to valley height was $64.0 \pm 24.0 \mu \mathrm{m}$. The spacing between stripes was $165.1 \pm 2.7 \mu \mathrm{m}, 10 \%$ larger than the nominal plate stripe width. The aluminum thin films were measured to be between 30 and $50 \mathrm{~nm}$ thick with $4.5 \pm 0.7 \mathrm{~nm}$ RMS surface roughness over a $3.24 \times 10^{-8} \mathrm{~m}^{2}$ and a sheet resistance of $1.59 \Omega . \square^{-1}$. 


\section{Discussion}

The oil thickness found by the thermal model is in the order of thickness that is deposited using flexography printing. Other oil deposition techniques would have to have their deposited thickness matched with the thermal energy deposited, the evaporation view factor and web speed can also be manipulated to ensure the correct balance of oil volume and radiative heating [20]. When it is vaporized from ambient temperature, the oil temperature change is only $42.5 \mathrm{~K}$ which is suitable for organic materials. It should be noted that the volume of oil required is small and so vacuum system contamination is minimal. The main source of error in the required liquid oil thickness value that was modelled is the determination of the particular oil's physical properties which can be limited in sales literature. The emissivity estimate can also vary with oil thickness.

The optical image of the metal pattern shows this technique has adequate edge roughness to be used in functional devices. Further quantitative image analysis has shown that at optimal printing pressure and velocity the peak-valley heights can be reduced below half the stripe spacing meaning critical shorting defects will be avoided, if the metal thin film pattern were to be applied to transistor contacts. The pattern resolution used here is not at the limit of this technique, which can achieve 10's of microns with even less rough line edges. However the challenge is engineering this process on large scale with such precision. Evidently the edge roughness is a result of the liquid oil on the substrate not a masking oil vapor, which would lead to a blurring and thinning of the deposition at the pattern's edges. This could imply that the aluminum deposition is not masked by the vapor pressure, as assumed above, but instead by the liquid oil on the substrate, such that there is condensation of the $\mathrm{Al}$ onto the surface of the liquid oil. In this case the latent heat of fusion of the deposited metal would also drive the thermal lift off process. This energy is 
approximately an order of magnitude greater than the radiative energy and so would require 10 times the amount of oil for effective masking which is larger than the maximum that could be transferred in this system. The implication, therefore, is that the oil vaporization is the dominant masking mechanism in the thermal lift off process, but that as this is simultaneous to the metal deposition the metal pattern adopts the form of the liquid oil still present on the substrate. In other words the oil vapor pressure prevents most aluminum deposition but as the liquid is also present on the substrate, its boundaries define the metal thin film pattern. The excess of deposited oil, compared to the amount of oil removed in the thermal lift process, was estimated to be within an order of magnitude which is valid for the assumptions made, but the excess estimated does not preclude some condensation of the eit Al vapor onto the liquid oil. This said, it is difficult to directly probe the oil's behavior in vacuum. However it can be concluded that the oil vapor cloud thickness would not influence the printed pattern quality, only the amount of deposition.

This thermal lift off description includes several assumptions. Firstly it is assumed that the process is adiabatic, besides the thermal radiation and vaporization of the oil. This is valid as the oil is within a vacuum with no conduction and negligible radiative pathways to lose thermal energy. The interface with the substrate could be considered, but the thermal conductivities would be difficult to ascertain and minimal. Secondly the oil's properties are assumed constant throughout. The oil viscosity could decrease as it is heated and the pattern distortion could be seen were this to lead to spreading or dewetting. Initial experiments did indicate some oil spreading ('dot gain') at ambient temperatures, there is little spreading time within the metallization zone but the time between oil pattern deposition and metallization is an important factor along with impression spreading effects. 
Finally, the oil temperature change is only $42.5 \mathrm{~K}$ which would not lead to large changes in viscosity.

The roughness and electrical characteristics of the patterned aluminum is equivalent to aluminum thin film coatings [21]. This makes the selective metallization technique attractive, as some ambient solution based printing methods, such as silver inks, do not achieve this conductivity with the same thickness and the printed solute can be quite rough [22,23]. Furthermore they require more chemical control, for solvent orthogonality, and use costly materials. This highlights the advantage of using direct in-vacuum deposition over solution processed techniques. 


\section{Conclusion}

The selective metallization process is a versatile technique for creating high quality metal patterns at process temperatures, modelled to be $62.5^{\circ} \mathrm{C}$, which are generally compatible with functional materials. In this paper a thermal model for calculating the thickness of oil on the substrate for effective masking of the oil was described and critiqued. The model suggests that printed oil a few microns thick would be adequate, although were some of the latent heat of fusion of the metal vapor condensing directly onto the liquid oil contributing to vaporization, the thickness would need to be greater. The estimated value of $1.63 \pm 0.31 \mu \mathrm{m}$ is based on the specific system geometry and view factors, and in a real world processing environment the patterning process should provide a thicker oil layer as complete masking is more vital than complete oil removal, which can be performed in post processing or remain with negligible effects.[24] It is assumed that the oil vapor has the largest masking effect but as the thermal lift off and metal deposition processes are simultaneous the metal pattern edges reflect the liquid oil's bounds. Hence the engineering challenge of reducing the line widths is limited most by controlling the liquid deposition process. That said the metal thin film properties are very attractive, compared to solvent based equivalents. The sheet resistance and roughness, $1.59 \Omega . \square^{-1}$ and $3.24 \times 10^{-8} \mathrm{~m}^{2}$ respectively, particularly set this high-throughput process apart as a promising technique for creating cost effective flexible electronics and organic devices. 


\section{Acknowledgements}

The authors would like to thank the Engineering and Physical Sciences Research Council for financial support.

Data associated with this publication can be found at XXX. (to be completed on acceptance) 


\section{References}

[1] N.J. Copeland, A. Jack, L. Harland, In-Register In-Vacuum Pattern Printing - From Wish to Reality, in: 52nd Annu. Tech. Conf. Proc., Society of Vacuum Coaters, Santa Clara, CA, 2009: p. 712.

[2] C.A. Bishop, Vacuum deposition onto webs, films, and foils, 2nd ed., William Andrew/Elsevier, Amsterdam; London, 2011.

[3] K. Nagatomi, T. Kajiwara, H. Ookuma, R. Tachibana, Process for producing metallized plastic film, US4832983 A, 1989.

[4] SelectMet ${ }^{\circledR} \quad$ - $\quad$ Overview, BOBST. http://www.bobst.com/usen/products/equipments/overview/machine/selectmet/\#.V7q6 4vkrKHs (accessed August 22, 2016).

[5] R. Parashkov, E. Becker, T. Riedl, H. Johannes, W. Kowalsky, Large Area Electronics Using Printing Methods, Proc. IEEE. 93 (2005) 1321-1329. doi:10.1109/JPROC.2005.850304.

[6] Gerard Cummins, Marc P.Y. Desmulliez, Inkjet printing of conductive materials: a review, Circuit World. 38 (2012) 193-213. doi:10.1108/03056121211280413.

[7] Søndergaard, Roar R, M. Hösel, F.C. Krebs, Roll-to-Roll fabrication of large area functional organic materials, J. Polym. Sci. Part B Polym. Phys. 51 (2013) 16-34. doi:10.1002/polb.23192.

[8] C.A. Bishop, Ink Jet for in vacuum pattern printing and deposition - a look at the feasibility, in: AIMCAL 2006 Fall Tech. Conf., 2006.

[9] S. Fujimoto, Patterning and high rate vacuum deposition on the super thin film, in: AIMCAL 2007 Fall Tech. Conf., 2007.

[10] H. Kipphan, ed., Handbook of print media: technologies and production methods, Springer, Berlin; New York, 2001. doi:10.1007/978-3-540-29900-4.

[11] A. Pagani, P. Raugei, F. Rimediotti, Patterned Metallized Films: State-of-the-art and Growth Opportunities, in: 50th Annu. Tech. Conf. Proc., Society of Vacuum Coaters, 2007: pp. 757-761.

[12] Krytox® Lubricants - By DuPont, (n.d.). http://www.krytox-lubricants.com/ (accessed April 14, 2017).

[13] D.R. Lide, CRC Handbook of Chemistry and Physics, 84th Edition, CRC Press, 2003.

[14] J.N. Lee, C. Park, G.M. Whitesides, Solvent Compatibility of Poly(dimethylsiloxane)Based Microfluidic Devices, Anal. Chem. 75 (2003) 6544-6554. doi:10.1021/ac0346712.

[15] R. Bickford, Analyze_Stripes ImageJ Plugin, 2013.

[16] C.A. Bishop, Roll-to-Roll Vacuum Deposition of Barrier Coatings, John Wiley \& Sons, 2015. doi:10.1002/9781118946183.

[17] Emissivity Table, Optotherm Therm. Imaging. (2016). http://www.optotherm.com/emiss-table.htm (accessed April 10, 2017).

[18] R.E. Honig, D.A. Kramer, Vapor Pressure Data for the Solid and Liquid Elements, RCA Laboratories, David Sarnoff Research Center, 1969.

[19] E.R. Booser, CRC Handbook of Lubrication and Tribology, Volume III: Monitoring, Materials, Synthetic Lubricants, and Applications, CRC Press, 1993.

[20] G. Comini, S. Savino, N. Magriotis, S. Muratori, Thermal modeling of vacuum web coating, Appl. Therm. Eng. $27 \quad$ (2007) 611-618. doi:10.1016/j.applthermaleng.2006.05.026.

[21] P. Quintana, A.I. Oliva, O. Ceh, J.E. Corona, M. Aguilar, Thickness effects on aluminum thin films, Superf. Vacío. 9 (1999) 280-282. 
[22] R. Venkata Krishna Rao, K. Venkata Abhinav, P.S. Karthik, S.P. Singh, Conductive silver inks and their applications in printed and flexible electronics, RSC Adv. 5 (2015) 77760-77790. doi:10.1039/C5RA12013F.

[23] D.J. Finn, M. Lotya, J.N. Coleman, Inkjet printing of silver nanowire networks, ACS Appl. Mater. Interfaces. 7 (2015) 9254-9261. doi:10.1021/acsami.5b01875.

[24] F. Rimediotti, Process \& Equipment for Specialty Web Products, in: AIMCAL, 2005. http://www.aimcal.org/2005-usa.html (accessed August 29, 2017). 


\section{List of Figure Captions}

Figure 1: Selective metallization process, showing in-vacuum flexography patterning of a sacrificial oil onto a substrate which passes over the metallization source to create a metal pattern and vaporize the oil simultaneously. The magnified view is a schematic of the phases present in the metallization zone. The schematic does not include the inking system of the flexography relief plate see reference [10] for more information about flexography systems.

Figure 2: Optical micrograph of an example of the printed aluminum thin films (dark gray) on PET. Image analysis [15] was used to find the patterns spacing and edge roughness. 
Figures (number below)

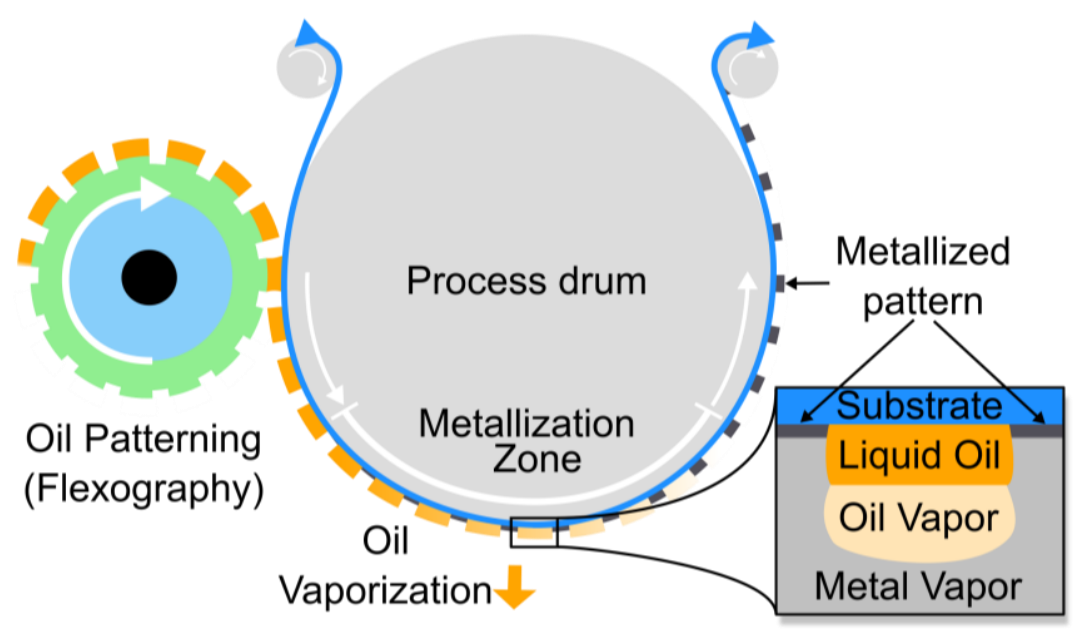

Radiation and vapor from metallization

source

1

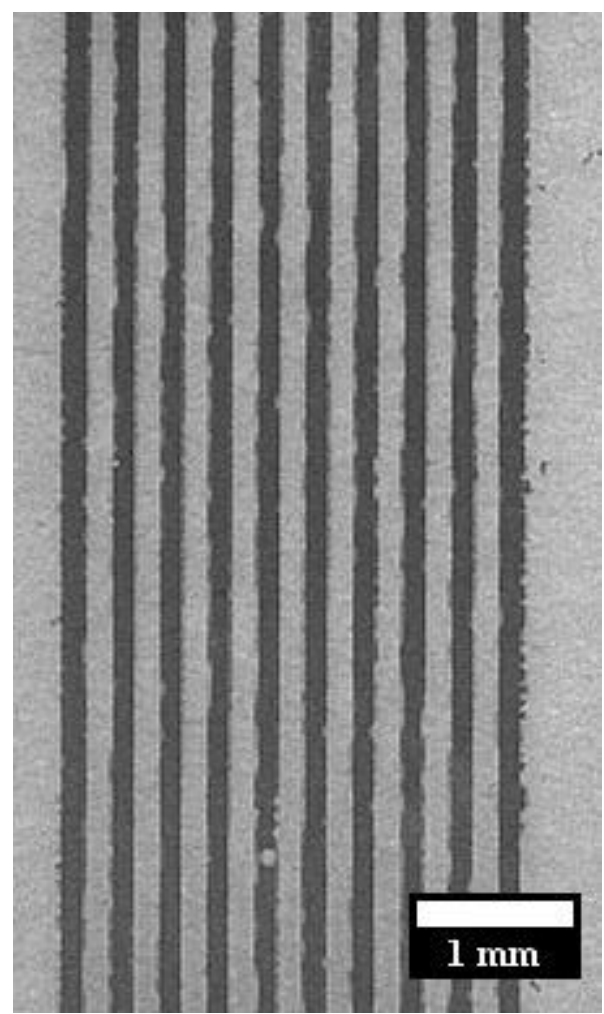

